Letters to the Editor

\title{
Group Separation of Ribonucleosides and Deoxyribonucleosides on a New Ceramic Titania Column
}

\author{
Masahiro Kawahara, Hiroshi Nakamura and Terumi Nakajima \\ Faculty of Pharmaceutical Sciences, University of Tokyo, Hongo, Tokyo 113, Japan
}

Keywords Titania, new ceramics, group separation, ribonucleoside, deoxyribonucleoside, high performance liquid chromatography

The adsorption properties of new ceramics titania (titanium dioxide, $\mathrm{TiO}_{2}$ ) as a column packing material for high performance liquid chromatography (HPLC) were investigated in our recent studies. ${ }^{1,2}$ The adsorption mechanism of titania was found to be different from that of silica gel; the titania adsorbs acidic compounds rather than basic compounds, while silica gel adsorbs basic compounds. ${ }^{2}$ In addition, titania possessed high resistance to both alkaline and acidic eluents. ${ }^{2}$ Therefore, a further attempt has been made to apply this titania column to the separation of various biogenic substances employing hydrophilic solvent systems. Among numerous biogenic substances tested, the titania column showed a peculiar property for nucleosides. This communication deals with the rapid group separation of ribonucleosides and deoxyribonucleosides on a titania column.

\section{Experimental}

\section{Chemicals}

Reagents and solvents were of guaranteed grade. Water was purified by Milli-Q reagent water system (Millipore, Bedford, MA, U.S.A.). Ribonucleosides (adenosine, guanosine, cytidine, uridine) and deoxyribonucleosides (thymidine, 2'-deoxyadenosine, 2'-deoxyguanosine, 2'-deoxycytidine, $2^{\prime}$-deoxyuridine) were purchased from Sigma Chemical Co., Ltd. (St. Louis, MO, U.S.A.).

\section{HPLC system}

Trirotar-VI (Jasco, Tokyo, Japan) HPLC system was used as described previously.' Titania microparticulates (Titania microbeads, $5-15 \mu \mathrm{m}$ ) from Catalysts \& Chemicals Ind. Co., Ltd. (Tokyo, Japan) were packed in a stainless steel tube ( $4 \mathrm{~mm}$ i.d. $\times 100 \mathrm{~mm}$ ) by using a slurry packing method ${ }^{2}$, and washed with $30 \mathrm{ml}$ of $10 \mathrm{mM}$ sodium hydroxide solution prior to use.

\section{Results and Discussion}

The titania acts predominantly as an adsorbent with negligible ion-exchange ability, as reported in our previous paper. ${ }^{2}$ The authors attempted the separation of a mixture of ribonucleosides and deoxyribonucleosides with a gradient elution using acetonitrile and $50 \mathrm{mM}$ borate buffer (pH 7.5). Deoxyribonucleosides (thymidine, deoxyuridine, deoxyguanosine, deoxycytidine, deoxyadenosine) were eluted near the front, while

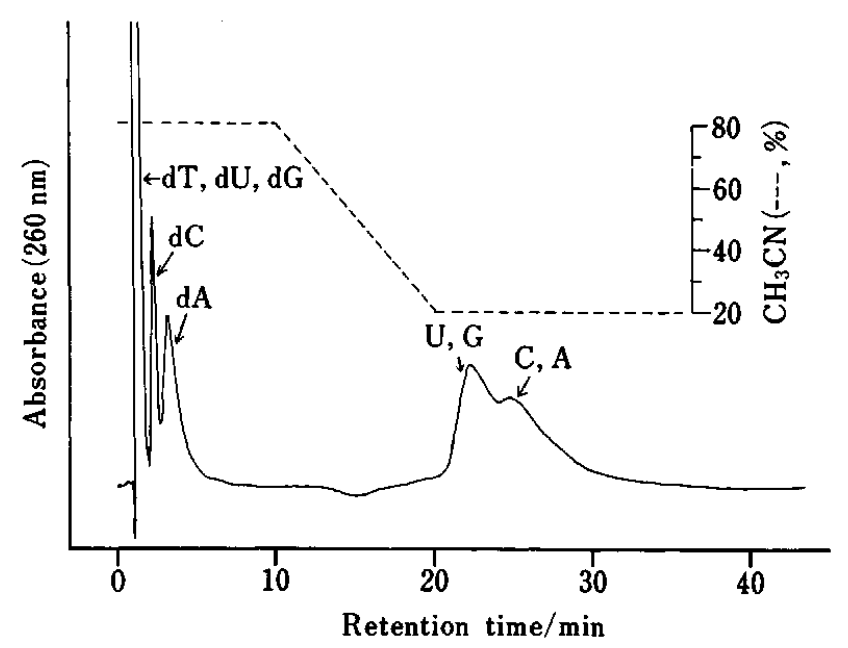

Fig. 1 Group separation of ribonucleosides and deoxyribonucleosides with a gradient elution. Column, titania $(4 \mathrm{~mm}$ i.d. $\times 100 \mathrm{~mm}$ ); Eluent $A$, acetonitrile- $50 \mathrm{mM}$ borate buffer $(\mathrm{pH} 7.5)=80 ; 20, \mathrm{v} / \mathrm{v}$; Eluent $\mathrm{B}$, acetonitrile- $50 \mathrm{mM}$ borate buffer $(\mathrm{pH} 7.5)=20: 80, \mathrm{v} / \mathrm{v}$; elution program: an isocratic elution with Eluent $A$ for $10 \mathrm{~min}$, and then a linear gradient from Eluent $A$ to Eluent $B$ in $10 \mathrm{~min}$; flow rate, $1 \mathrm{ml} / \mathrm{min}$; column temperature, $40^{\circ} \mathrm{C}$; peak identity: $\mathrm{dT}$, thymidine; dU, 2'-deoxyuridine; dG, 2'-deoxyguanosine; dC, 2'-deoxycytidine; dA, 2'-deoxyadenosine; U, uridine; G, guanosine; $\mathrm{C}$, cytidine; $\mathrm{A}$, adenosine; injected amount, $5 \mathrm{nmol}$ each. 


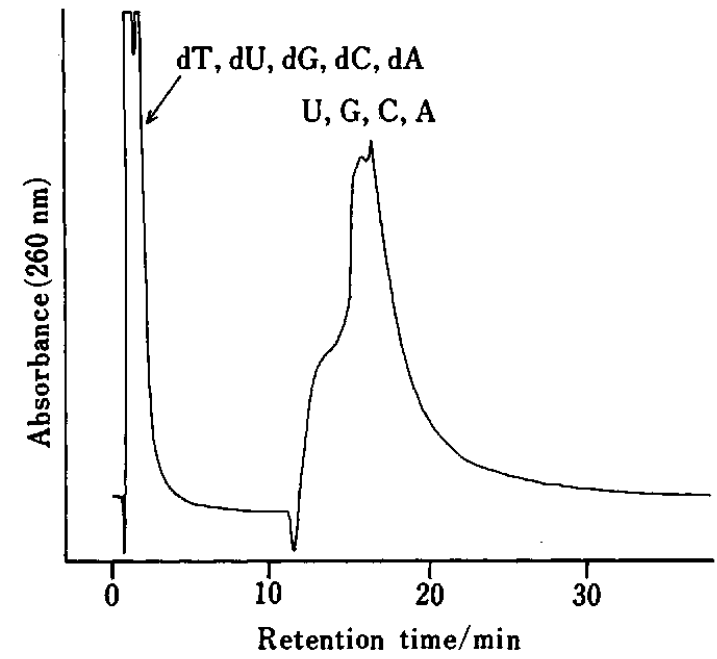

Fig. 2 Group separation of ribonucleosides and deoxyribonucleosides with a stepwise elution. Eluent $\mathrm{A}, 2 \mathrm{mM}$ Tris- $\mathrm{HCl}$ buffer ( $\mathrm{pH} \mathrm{7.0);} \mathrm{Eluent} \mathrm{B,} 100 \mathrm{mM}$ Tris-HCl buffer (pH 7.0); elution program: an isocratic elution with Eluent $A$ for $8 \mathrm{~min}$, and then the stepwise elution with Eluent B. Other conditions were the same as in Fig. 1.

nucleosides (uridine, guanosine, cytidine, adenosine) eluted afterwards as broad peaks (Fig. 1). This characteristic of titania column was almost the same in an isocratic elution of $5 \mathrm{mM}$ Tris- $\mathrm{HCl}$ buffer ( $\mathrm{pH} 7.0$ ), i.e., deoxyribonucleosides were eluted in front, while ribonucleosides were adsorbed on the column and eluted later as broad bands. It is noteworthy that the behaviors of ribonucleosides and deoxyribonucleosides on titania are obviously different in spite of the similarity in their structures, except in the 2' position of the sugar moieties. Consequently, a rapid group separation of ribonucleosides and deoxyribonucleosides may be achieved by the stepwise elution conditions as shown in Fig. 2. Some kinds of boronate gels ${ }^{3-5}$ have been employed for both the group separation ${ }^{3}$ of ribonucleosides and deoxyribonucleosides and preconcentration ${ }^{4}$ of compounds having a vicinal hydroxy group. Our method may be applied for these purposes in the light of its rapidity and simplicity. We have also observed that nucleotides are strongly retained on the titania column and that they do not interfere with the separation of nucleosides. Consequently, the titania column is expected to become a tool as useful as the alumina column ${ }^{6}$, in a two-dimensional HPLC separation system in combination with a different separation mode such as reversed phase HPLC on an ODS silica.

The present work was partially supported by a Grant-inAid (HN, No. 63571018) from the Ministry of Education, Science and Culture of Japan.

\section{References}

1. M. Kawahara, H. Nakamura and T. Nakajima, Anal. Sci., 4, 671 (1988).

2. M. Kawahara, H. Nakamura and T. Nakajima, Anal. Sci., 5, 485 (1989).

3. M. Glad and S. Ohlson, J. Chromatogr., 200, 254 (1980).

4. E. H. Pfádenhauer and Sun-De Tong, J. Chromatogr., 162, 585 (1979).

5. S. Hjérten and D. Yang, J. Chromatogr., 316, 301 (1984).

6. H. Tanaka, M. Koike and T. Nakajima, Anal. Sci., 2, 385 (1986).

(Received September 16, 1989)

(Accepted October 5, 1989) 